\title{
Genome-wide compound heterozygote analysis highlights alleles associated with adult height in Europeans
}

\author{
Kaiyin Zhong ${ }^{1}$ - Gu Zhu $^{2} \cdot$ Xiaoxi Jing ${ }^{3,4}$ - A. Emile J. Hendriks ${ }^{5,6}$ • \\ Sten L. S. Drop ${ }^{5}$ M. Arfan Ikram ${ }^{7} \cdot$ Scott Gordon $^{2} \cdot$ Changqing Zeng 3 • \\ Andre G. Uitterlinden ${ }^{7,8} \cdot$ Nicholas G. Martin $^{2} \cdot$ Fan Liu $^{1,3,4}{ }^{(\mathbb{B}} \cdot$ Manfred Kayser $^{1}$
}

Received: 13 March 2017 / Accepted: 26 August 2017 / Published online: 18 September 2017

(C) The Author(s) 2017. This article is an open access publication

\begin{abstract}
Adult height is the most widely genetically studied common trait in humans; however, the trait variance explainable by currently known height-associated single nucleotide polymorphisms (SNPs) identified from the previous genome-wide association studies (GWAS) is yet far from complete given the high heritability of this complex trait. To exam if compound heterozygotes $(\mathrm{CH})$ may explain extra height variance, we conducted a genome-wide analysis to screen for $\mathrm{CH}$ in association with adult height in 10,631 Dutch Europeans enriched with extremely tall people, using our recently developed method implemented in the software package CollapsABEL. The analysis identified six regions (3q23, 5q35.1, 6p21.31, 6p21.33, 7q21.2, and 9p24.3), where multiple pairs of SNPs as $\mathrm{CH}$ showed genome-wide significant association with height $\left(P<1.67 \times 10^{-10}\right)$. Of those, 9 p24.3 represents a novel region influencing adult height, whereas the others have been highlighted in the
\end{abstract}

Fan Liu and Manfred Kayser contributed equally to this study.

Electronic supplementary material The online version of this article (doi:10.1007/s00439-017-1842-3) contains supplementary material, which is available to authorized users.

Fan Liu

liufan@big.ac.cn

$\bowtie$ Manfred Kayser

m.kayser@erasmusmc.nl

1 Department of Genetic Identification, Erasmus MC University Medical Center Rotterdam, Rotterdam, The Netherlands

2 Queensland Institute of Medical Research, Brisbane 4029, Australia

3 Key Laboratory of Genomic and Precision Medicine, Beijing Institute of Genomics, Chinese Academy of Sciences, Beijing, China previous GWAS on height based on analysis of individual SNPs. A replication analysis in 4080 Australians of European ancestry confirmed the significant $\mathrm{CH}$-like association at 9p24.3 $(P<0.05)$. Together, the collapsed genotypes at these six loci explained $2.51 \%$ of the height variance (after adjusting for sex and age), compared with $3.23 \%$ explained by the 14 top-associated SNPs at 14 loci identified by traditional GWAS in the same data set $\left(P<5 \times 10^{-8}\right)$. Overall, our study empirically demonstrates that $\mathrm{CH}$ plays an important role in adult height and may explain a proportion of its "missing heritability". Moreover, our findings raise promising expectations for other highly polygenic complex traits to explain missing heritability identifiable through $\mathrm{CH}$-like associations.

\section{Introduction}

Human adult height is highly influenced by genetic factors with an estimated heritability of up to $80 \%$ (Carmichael and McGue 1995; Nielen et al. 2001; Silventoinen et al. 2003, 2008). A number of large-scale GWASs (Estrada et al. 2009;

4 University of Chinese Academy of Sciences, Beijing, China

5 Division of Endocrinology, Department of Pediatrics, Sophia Children's Hospital, Erasmus MC University Medical Center Rotterdam, Rotterdam, The Netherlands

6 Department of Pediatrics, University of Cambridge, Cambridge, UK

7 Department of Internal Medicine, Erasmus MC University Medical Center Rotterdam, Rotterdam, The Netherlands

8 Department of Epidemiology, Erasmus MC University Medical Center Rotterdam, Rotterdam, The Netherlands 
Liu et al. 2014; Wood et al. 2014; Yang et al. 2010), carried out to explore the genetic architecture underlying the variation in height, have successfully identified at close to 700 common single nucleotide polymorphisms (SNPs) (Wood et al. 2014), and more recently 83 low-frequency (Marouli et al. 2017) variants, showing genome-wide significant association with height. However, these SNPs together can only explain about 21.6\% (Marouli et al. 2017) of the phenotypic variance in the study populations (combined sample size 711,428 individuals) due to the extremely small effect sizes for most associated SNPs. Large fractions of the "missing heritability" are also known to occur in a wide range of human complex traits and common disorders (Keller et al. 2012; Ridge et al. 2013; Tomlinson et al. 2011). Missing heritability represents the central challenge in understanding the genetics of common traits in humans, with consequences for future applications in different fields such as precision medicine and forensic genetics.

Aside from epigenetic factors, there are two mutually compatible leading theories for the major sources of this missing heritability. The first theory emphasizes that there may exist a large body of rare or low-frequency DNA variants, each with moderate-to-large effects (Bodmer and Bonilla 2008). Such variants are not detectable due to low statistical power, low array coverage, and low imputation quality for rare variants (Yang et al. 2015). Consequently, the key to solving the "missing heritability" puzzle is to scale up sample sizes together with genome-sequencing-based techniques, which are both time-consuming and expensive. For example, a very recent exome chip study of height carried out by the GIANT Consortium identified 32 extremely rare (MAF < 0.01 ) height-associated variants, some with much larger effects than previously seen for common SNPs, using a data set consisting of 840,552 individuals (Marouli et al. 2017). The second theory stresses that variation in most quantitative phenotypes must result at least in part from multifactorial genetic perturbation of highly dynamic, interconnected biochemical networks (Mackay 2014), while the traditional association studies focus on additive effects of individual genes (Carlborg and Haley 2004; Manolio et al. 2009). The challenge here is that the abundance of complex forms of genetic interactions will be hard to detect due to the problem of combinatorial complexity that demands largescale computational power.

Compound heterozygotes $(\mathrm{CH})$ in classical genetics refer to two different mutations at a particular gene, one on each chromosome-both typically rare and deleterious (Schaaf et al. 2011), together cause an autosomal recessive trait. $\mathrm{CH}$ has been found in nearly all autosomal recessive disorders, where multiple mutations (i.e., different alleles) can affect the function of the gene product and lead to disease (Bezzina et al. 2003; Krude et al. 1998; Liu et al. 2011; Whittock et al. 2002), and therefore, a common genetic mechanism is assumed. Considering that trait-associated DNA variants identified from GWAS are typically non-coding due to the design of the SNP microarrays used, and can thus be involved in the regulation of gene expression (Visser et al. 2012, 2014, 2015), it is reasonable to expect that interactions involving non-coding DNA variants can also be highly relevant in the genetics of complex traits. Therefore, we recently proposed relaxed forms of $\mathrm{CH}$, in which genetic variants physically close to one another [and in linkage disequilibrium (LD) to one another] are likely involved in a wide range of human polygenic traits and may present an important source of the "missing heritability" (Liu et al. 2016; Zhong et al. 2016a). Accordingly, we had developed a generalized collapsed double heterozygosity (GCDH) test to screen for $\mathrm{CH}-$ like association in GWASs. This method is implemented in the publically available R package CollapsABEL (Zhong et al. 2016a). Recently, we demonstrated that this approach was able to detect new genetic loci associated with the skin condition actinic keratosis via genome-wide GCDH analysis (Zhong et al. 2016b).

In the current study, we investigate if CH-like SNP interactions are associated with adult height in Europeans, and if so, to which extent they contribute to explaining the "missing heritability" previously noted for height with the conventional GWAS approach. For this, we used CollapsABEL to carry out genome-wide GCDH analysis to previously established genome-wide SNP array data of 10,361 Dutch Europeans from the Rotterdam Study (RS), including 770 additional Dutch Europeans of extremely tall stature (Liu et al. 2014), and used data from 4080 unrelated individuals of European origin from the Queensland Institute of Medical Research (QIMR) as a replication data set.

\section{Results}

Characteristics of the study populations are listed in Online Resource 1. A conventional GWAS in 10,361 Dutch Europeans from the RS including 770 extremely tall Dutch Europeans identified 101 SNPs from 14 loci (Fig. 1a and Online Resource 2), showing genome-wide significant association with height $\left(P<5 \times 10^{-8}\right)$. The top-associated SNPs at each locus were rs12485899 from 3p22.1 (ZNF621, $\left.P=3.50 \times 10^{-9}, \beta=-0.57\right)$, rs9838625 from 3q23 (ZBTB38, $\left.P=2.71 \times 10^{-10}, \beta=0.62\right)$, rs1265097 from 6p21.33 (PSORS1C1, $P=5.39 \times 10^{-9}$, $\beta=-0.89$ ), rs 2780226 from 6p21.31 (GRM4/HMGAl, $\left.P=4.26 \times 10^{-8}, \beta=0.92\right)$, rs7741741 from $6 \mathrm{q} 24.1$ (GPR126, $\left.P=1.72 \times 10^{-8}, \beta=-0.6\right)$, rs4272 from $7 \mathrm{q} 21.2$ (CDK6, $\left.P=4.23 \times 10^{-9}, \beta=0.68\right)$, rs6984782 from $8 \mathrm{q} 12.1$ (CHCHD7, $\left.P=1.80 \times 10^{-9}, \beta=-0.94\right)$, rs 4931222 from 12p11.22 (TMTCl, $P=3.05 \times 10^{-8}, \beta=-1.36$ ), rs 1038196 from 12q14.3 (HMGA2, $\left.P=3.86 \times 10^{-8}, \beta=-0.53\right)$, 

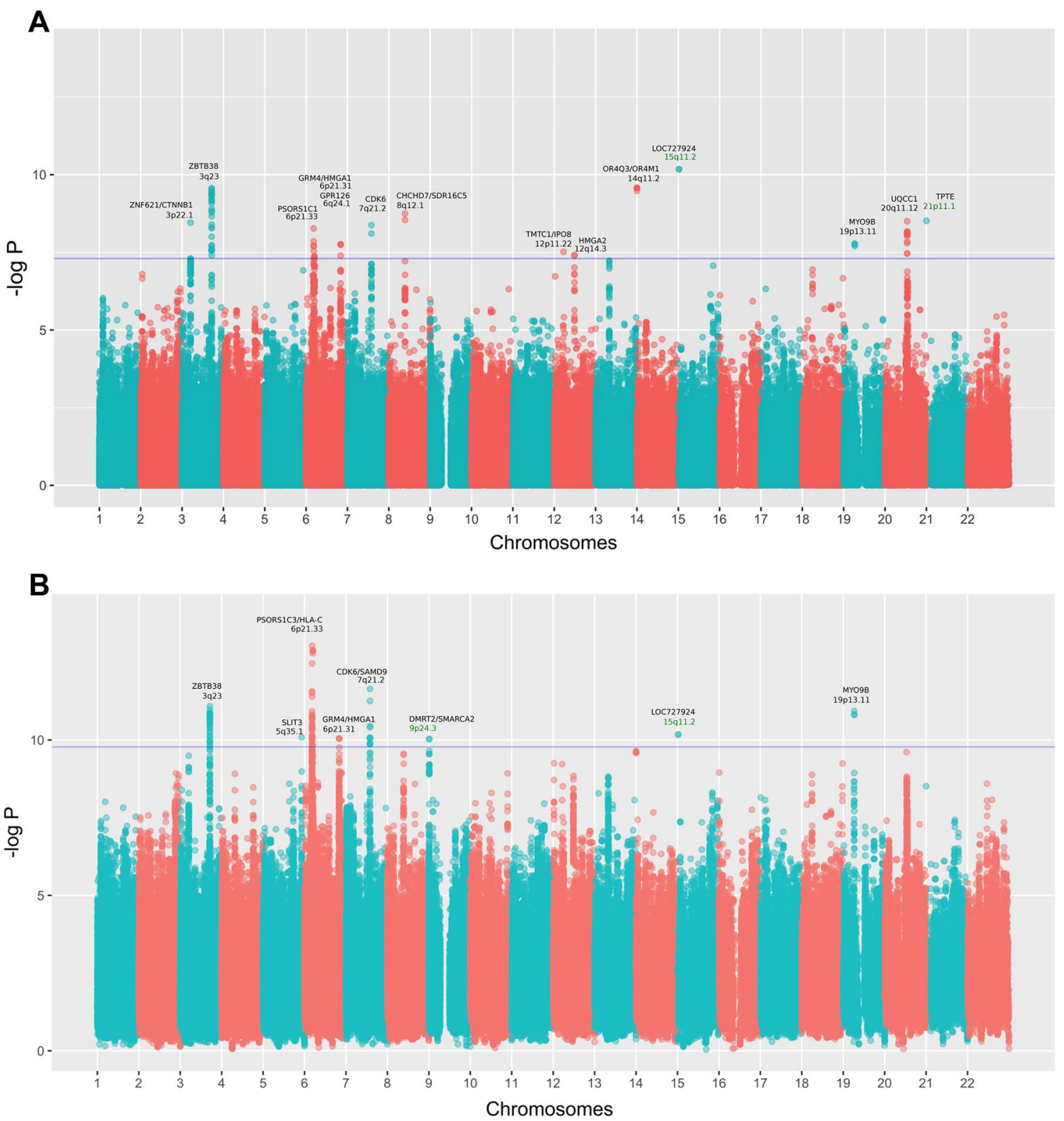

Fig. 1 Manhattan plots from RS. Minus $\log$ of $P$ value is represented by blue and red dots and grouped by chromosome. a Manhattan plot for single-SNP GWAS. The horizontal line $\left(-\log 5 \times 10^{-8}\right)$ indicates threshold of genome-wide significance. b Manhattan plot for GCDH scan, the horizontal line $\left(-\log 1.67 \times 10^{-10}\right)$ indicates threshold of GCDH genome-wide significance. Loci are annotated in green if they have not been reported before with genome-wide height association by the GIANT consortium (Wood et al. 2014)

(Wood et al. 2014). Note that the test statistics from our conventional GWAS slightly deviated from the expected distribution under the null hypothesis of no association $(\lambda=1.05$, Fig. 2a). In contrast, the test statistics from a permutation analysis ( $k=5$, Fig. 2a) followed the expected distribution under the null tightly, suggesting that the significant signals are unlikely false positives due to residual population substructures.

We then conducted an exact replication analysis of these 14 SNPs in 4080 unrelated Australian Europeans from the Queensland Institute for Medical Research (QIMR). The two identified by the GIANT Consortium via traditional GWAS 

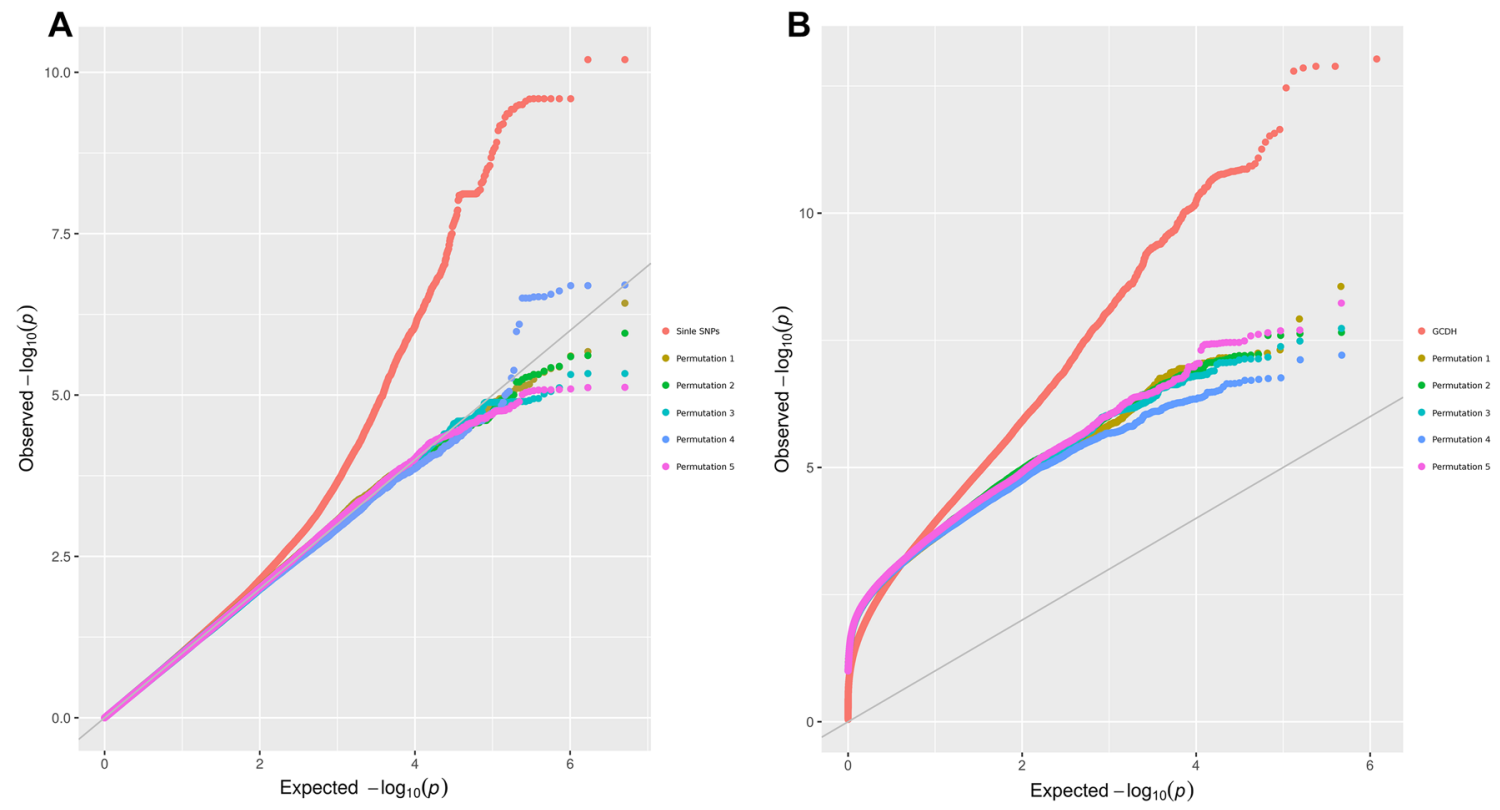

Fig. 2 QQ plots. a QQ plot of single-SNP $P$ values. b QQ plot of GCDH $P$ values. In both cases, $P$ values from five permutation analyses were also plotted to estimate the null distribution

novel loci which had very low MAF in QIMR (0.1-0.2\%) are thus not available for replication. For the remaining 12 SNPs, 10 showed the same allele effect direction as in the RS and 6 showed nominally significant association with height ( $P<0.05$, Online Resource 2), highlighting GRM4/HMGAl, GPR126, CDK6, HMGA2, MYO9B, and UQCC1 genes. The replication rate of the single SNPs was lower than expected from our power analysis, which showed a $>90 \%$ probability of replicating all the 14 SNPs (see Online Resource 3). The lower replication rate may be explained by multiple factors. For example, allelic heterogeneity has been previously noted as a frequent feature underlying the genetic architecture of adult (Allen et al. 2010). Notably, the novel loci 15q11.2 and 21p11.1 did not replicate because of the very low MAF $(0.1-0.2 \%)$ in QIMR.

We conducted a genome-wide screening for relaxed forms of compound or double heterozygotes that are potentially associated with adult height in the RS. This analysis was conducted using our recently developed software package CollapsABEL implementing a generalized collapsed double heterozygosity test (GCDH). The GCDH employs a generalized linear modeling framework to test the association between a pseudo-marker and a phenotype with or without covariates, where the pseudo-marker is collapsed from two physically close SNPs, as described in more details in the "Materials and methods". The test statistics from the GCDH genome screening (Fig. 2b) showed a large deviation $(\lambda=4.16)$ from the expected null of the conventional GWAS as the result of multiple testing from the sliding window approach implemented in CollapsABEL. This deviation is expected, because first, CollapsABEL only reports the most significant association signal per window, i.e., a problem of multiple testing of correlated SNPs, and second, a p-filter was applied to pre-remove SNPs with little or no marginal effect. Therefore, we conducted a permutation analysis to approximate the null empirically, i.e., repeating the full GCDH genome screening five times using permuted phenotype values, and considered the distribution of the resultant test statistics as an empirical null distribution (Fig. 2b). Although the number of permutation is small due to the computational burden, it is obvious that the GCDH results deviated substantially from the empirical null distribution, which is unlikely explained by false-positive findings (Fig. 2b). This GCDH screen provided genome-wide significant evidence for six genetic regions consisting of $\mathrm{CH}-$ like association signals from the collapsed pseudo-markers (3q23, 5q35.1, 6p21.31, 6p21.33, 7q21.2, and 9p24.3, $P<1.67 \times 10^{-10}$, see Fig. 1b; Table 1 and Online Resource 4). Notably, none of the individual SNPs in these regions showed genome-wide significant association in our conventional GWAS $\left(P>5 \times 10^{-8}\right)$. The top-associated pseudomarkers were collapsed from SNP pairs in these six regions, including rs9821337_rs6762826 from 3q23 (ZBTB38, $\left.P=9.59 \times 10^{-11}, \beta=0.62\right)$, rs1466947_rs17070997 from 5q35.1 (SLIT3, $\left.\mathrm{P}=8.21 \times 10^{-11}, \beta=-0.78\right)$, rs 1776897 rs2744977 from 6p21.31 (GRM4/HMGA1/C6orf106, $\left.P=1.61 \times 10^{-13}, \beta=0.78\right)$, rs6904669_rs1960278 from 6p21.33 (PSORSIC3/HLA-C, $P=9.32 \times 10^{-14}, \beta=-0.89$ ), rs7793983_rs17164894 from 7q21.2 (CDK6/SAMD9, $\left.P=3.56 \times 10^{-11}, \beta=0.87\right)$, and rs514779_rs10962274 from 


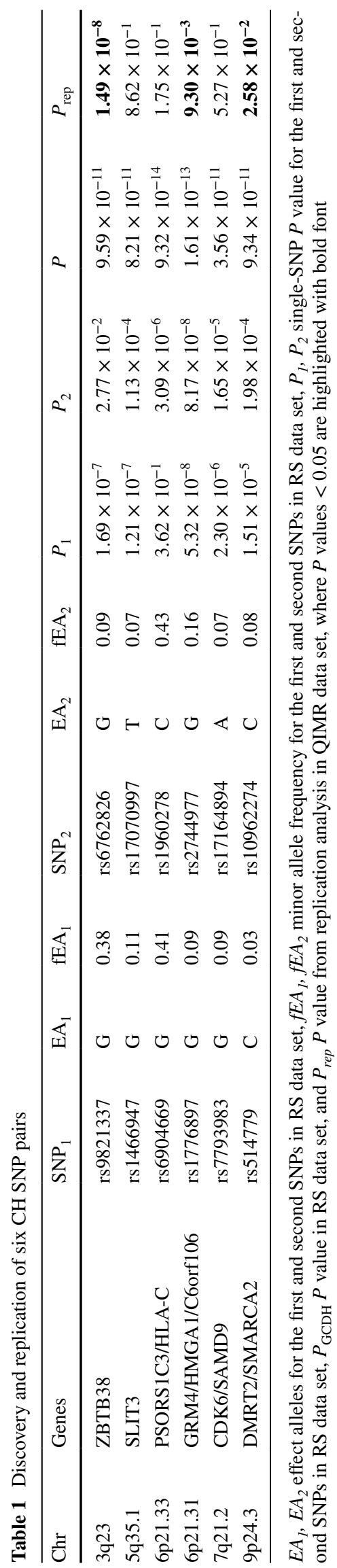

9p24.3 (DMRT2/SMARCA2, $\left.P=9.34 \times 10^{-11}, \beta=-0.98\right)$. One of the six loci, 9 p24.3 (see also the regional plot, as shown in Fig. 3), represents a novel finding, whereas the other five (see regional plots in Online Resource 5) had been previously described as influencing height variation by the GIANT Consortium from a large-scale conventional GWAS (Wood et al. 2014). Significant GCDH signals were detected at two additional loci, 15q11.2 and 19p13.11, but are potentially explained by strong single-SNP association effects rather than the effect of a pair of SNPs, since individual SNP analysis already detected those SNPs as genome-wide significant (rs1366870 and rs2217377 in 15q11.2 and rs11671774, rs2279008, and rs2279007 in 19p13.11, see Fig. 1).

We then conducted an exact replication analysis of these six SNP pairs in the QIMR data set. The newly identified locus 9p24.3 (rs514779_rs10962274, $P=0.026, \beta=-0.48$ ) as well as three previously known markers were replicated at nominal significance, i.e., rs9821337_rs6762826 from 3q23 $\left(P=1.49 \times 10^{-8}, \beta=0.72\right)$, rs $1776897 \_r s 2744977$ from 6p21.31 $(P=0.0093, \beta=0.37)$, rs17688839_rs17164894 from $7 \mathrm{q} 21.2(P=0.0019, \beta=0.44)$. All of these replicated $\beta$ values showed the same directions as those in the Dutch Europeans (Table 1).

A previous large-scale meta-analysis of height GWASs identified 697 SNPs at different loci independently contributing to the variation in adult height and 658 SNPs were available in our RS data set. The proportion of sex- and age-adjusted height variance explainable by these 658 SNPs in our data set was estimated at $19.13 \%$ by constructing a polygenic risk score based on the weighted allele sums of all 658 SNPs (Table 2). This polygenic score is referred to below as PRS658. A multivariable analysis further demonstrated that on top of the PRS658, the six top-associated pseudo-markers collapsed from six pairs of SNPs explained $2.51 \%$, and the 14 top-associated SNPs from our conventional GWAS explained $3.23 \%$ of the sex- and age-adjusted height variance (Table 2). For all six loci, the top-associated pseudo-marker explained a substantially higher proportion of the trait variance than individual SNPs of a pair, i.e., when any of the two SNPs was tested in a univariate model (Online Resource 6), and explained a higher or similar proportion of the trait variance than the two SNPs together, i.e., when both SNPs were included in a multiple regression model (Online Resource 6). This indicates that including the pseudo-markers in prediction modeling may not necessarily boost the prediction accuracy compared with the models including all pairs of SNPs in $\mathrm{CH}$. However, it should be emphasized that the use of the pseudo-marker provides a substantially higher power in detecting $\mathrm{CH}$-like association signals than the conventional GWAS.

Diplotype configuration inference supports the involvement of compound heterozygotes for three of the six pseudomarkers ( $3 \mathrm{q} 23,6 \mathrm{p} 21.33$, and $7 \mathrm{q} 21.2)$ and the involvement of 
Fig. 3 Regional Manhattan plot of GCDH $P$ values from 9p24.3

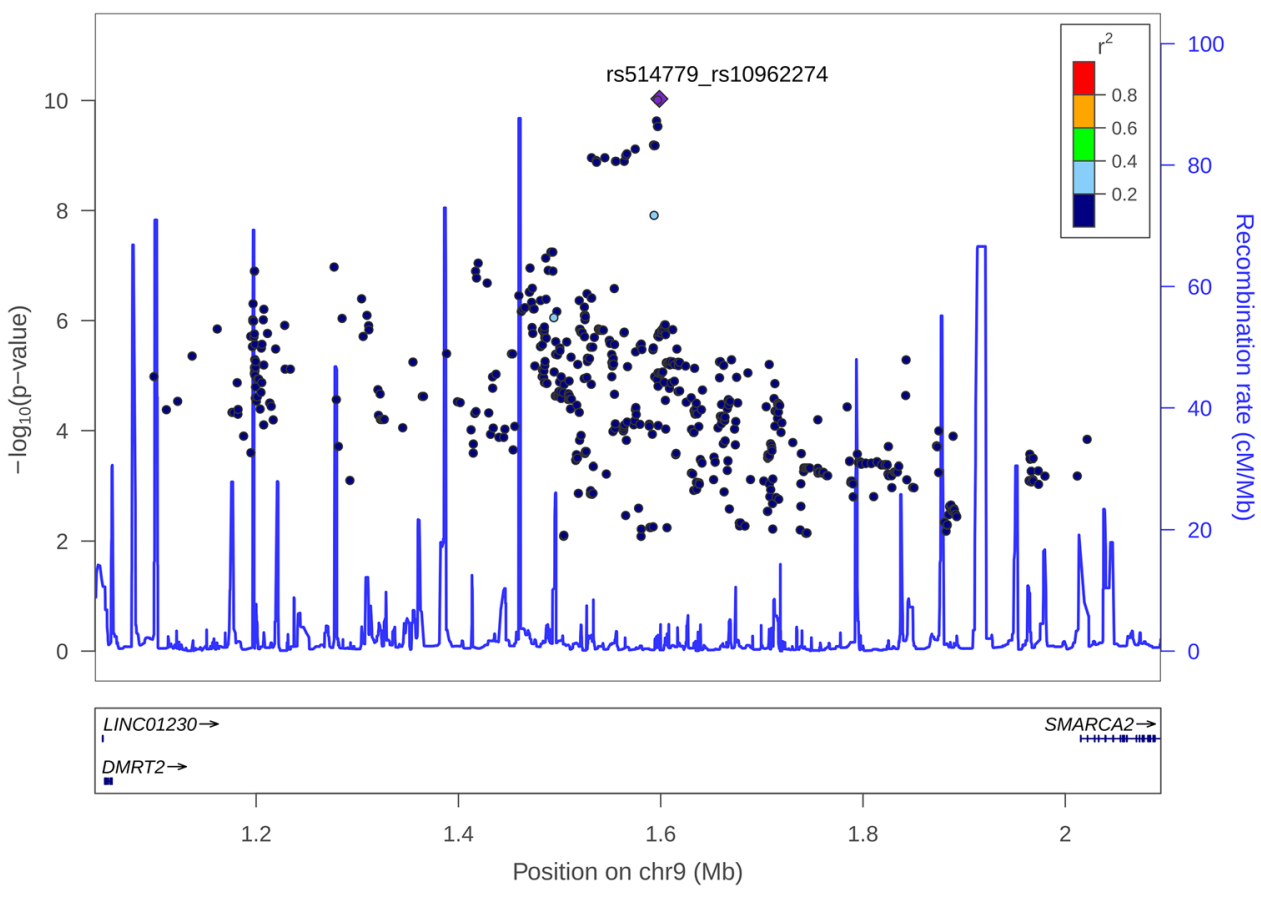

Table 2 Multivariable linear model including 14 single SNPs, 6 CH SNP pairs and the weighted allele sum of 658 SNPs previously reported to be associated with height as independent variables

\begin{tabular}{|c|c|c|c|c|}
\hline Variable & Cytoband & Gene & $-\log _{10} P$ & $R^{2}$ \\
\hline PRS658 & & & 945.12 & 19.13 \\
\hline rs10439884 & $21 \mathrm{p} 11.1$ & TPTE & 19.22 & 0.32 \\
\hline rs7159961 & $14 \mathrm{q} 11.2$ & OR4Q3 & 17.25 & 0.33 \\
\hline rs1366870 & $15 q 11.2$ & LOC727924 & 16.33 & 0.31 \\
\hline rs2279007 & 19p13.11 & MYO9B & 15.53 & 0.44 \\
\hline rs4931222 & $12 \mathrm{p} 11.22$ & TMTC1 & 13.10 & 0.29 \\
\hline rs12485899 & $3 \mathrm{p} 22.1$ & ZNF621 & 11.35 & 0.30 \\
\hline rs 4272 & $7 q 21.2$ & CDK6 & 4.99 & 0.28 \\
\hline rs6984782 & $8 \mathrm{q} 12.1$ & $\mathrm{CHCHD} 7$ & 3.33 & 0.26 \\
\hline rs7741741 & $6 \mathrm{q} 24.1$ & GPR126 & 3.25 & 0.30 \\
\hline rs1038196 & $12 q 14.3$ & HMGA2 & 2.10 & 0.23 \\
\hline rs9838625 & $3 q 23$ & ZBTB38 & 1.93 & 0.12 \\
\hline rs1265097 & $6 \mathrm{p} 21.33$ & PSORS1C1 & 1.89 & 0.04 \\
\hline rs6060355 & $20 q 11.22$ & UQCC1 & 1.38 & 0.01 \\
\hline rs2780226 & $6 \mathrm{p} 21.31$ & GRM4 & 0.90 & 0.01 \\
\hline rs514779_rs10962274 & $9 \mathrm{p} 24.3$ & DMRT2/SMARCA2 & 19.20 & 0.39 \\
\hline rs1466947_rs17070997 & $5 q 35.1$ & SLIT3 & 13.17 & 0.39 \\
\hline rs7793983_rs17164894 & $7 q 21.2$ & LOC101927497 & 11.65 & 0.50 \\
\hline rs6904669_rs1960278 & $6 \mathrm{p} 21.33$ & PSORS1C3/HLA-C & 10.39 & 0.48 \\
\hline rs1776897_rs2744977 & $6 \mathrm{p} 21.31$ & GRM4/HMGA1 & 4.02 & 0.39 \\
\hline rs9821337_rs6762826 & $3 q 23$ & ZBTB38 & 0.76 & 0.36 \\
\hline Total $1^{\mathrm{a}}$ & & & & 2.51 \\
\hline Total $2^{\mathrm{b}}$ & & & & 3.23 \\
\hline Total $3^{c}$ & & & & 24.87 \\
\hline
\end{tabular}

${ }^{\text {a }}$ Total percentage of variation in height explained by 6 CH SNPs

${ }^{\mathrm{b}}$ Total percentage of variation in height explained by 14 single SNPs

${ }^{c}$ Total percentage of variation in height explained by all independent variables in the model 
double (non-compound) heterozygotes (see Online Resource 7 for an illustration and double and compound heterozygosity) for the other three pseudo-markers (Online Resource 8).

\section{Discussion}

An empirical application of the GCDH approach (as implemented in CollapsABEL) to height identified novel $\mathrm{CH}$-like interactions and markers that are likely not identifiable by the conventional GWAS. With only a small fraction of the sample size of the GIANT study (Wood et al. 2014), we found $\mathrm{CH}$-like interactions among known height-related SNPs and one previously unknown marker, which in turn explained a sizable percentage of variance in height. It is, therefore, expected that an application of CollapsABEL to the full GIANT data set (Wood et al. 2014) will reveal many more $\mathrm{CH}$-like interactions which may include novel height loci not identifiable via standard single-SNP GWAS.

The two SNPs rs514779 and rs1853417 from the novel region 9p24.3 are located between the genes DMRT2 and SMARCA2. DMRT2 is a candidate gene for sex determination and is also involved in development of somites and muscle tissue in the embryo (Ottolenghi et al. 2000; Ounap et al. 2004; Seo 2007). Both functions may be important in determining adult height, although $D M R T 2$ has not been reported to be associated with height in the literature. SMARCA2 encodes a protein, that is, part of the ATP-dependent chromatin remodeling complex SNF/SWI, which is required for transcriptional activation of genes normally repressed by chromatin (Koga et al. 2009). The interaction between the transcription-regulator gene SMARCA2 and the development-related gene DMRT2 makes perfect sense, although it needs further functional study to be verified.

The other five loci highlighted via CH-like effects (3q23, 5q35.1, 6p21.31, 6p21.33, and 7q21.2) have all been previously identified via large-scale traditional single-SNP GWAS of the GIANT Consortium (Wood et al. 2014). However, the CH-like interactions of SNPs found here had not been reported before. These results indicate that $\mathrm{CH}$-like interactions among known loci may explain a sizable proportion of the phenotypic variance. The six best CH SNP pairs chosen from each significant locus accounted for $2.51 \%$, compared to $3.23 \%$ by the 14 single SNPs from RS and $19.13 \%$ by PRS658. This clearly demonstrates that phenotypic variance explained by genetic $\mathrm{CH}$-like interactions can be orthogonal to that attributed to single SNPs, a finding for height that is expected for other complex traits as well.

Notably, we have used an additive model, i.e., the alternative collapsing matrix (Zhong et al. 2016a), for genotype collapsing, which does not strictly differentiate between compound heterozygosity and non-compound double heterozygosity or any form of pairwise interaction between two variants showing an additive effect. As a consequence, the SNP pairs discovered cannot be expected to be all in $\mathrm{CH}$. According to our diplotype inference results (Online Resource 8), GCDH signals were confirmed in three (3q23, 6p21.33, and 7q21.2) of the six most significant pseudomarkers. This implies that the relaxed form of $\mathrm{CH}$ is an important mechanism among the possible interaction forms conforming to the additive model, although non-compound double heterozygosity and possibly other kinds of genetic interactions may also play a significant role. Besides the additive model, other possible models (collapsing matrices) representing more complex/unknown genetic mechanisms could be explored in the future, due to the design flexibility of CollapsABEL.

Compared to recent large-scale traditional single-SNP GWASs for height carried out by the GIANT Consortium, consisting of 253,288 (Wood et al. 2014) up to 381,625 (Marouli et al. 2017) samples, the current study is of relatively small sample size, which limits statistical power. In addition, the preprocessing of genotype data with a $P$ value filter, which removes all SNPs without a strong enough marginal effect, helped reducing computational burden, but also constrained the scope of discoverable interactions. However, the main goal of our study was to find out if and to what extent can the signals detected by CollapsABEL explain "missing heritability". To maximize statistical power in identifying novel $\mathrm{CH}$ variants, we included a set of 770 extremely tall individuals in the RS data set. It had been shown previously that the effects of height-associated common DNA variants are consistent with the predicted polygenic effects in extremely tall persons (Chan et al. 2011). This consistency is the basis for a successful replication in the QIMR data set which does not include extremely tall subjects. Our previous study (Liu et al. 2014) using the same RS data also confirmed that the genetics of tall stature is similar to that of normal height in humans, i.e., heritability is for a large part explained by many common variants with small effects and allelic heterogeneity is a frequent feature, where $\mathrm{CH}$ represents a common form of allelic heterogeneity.

In comparing the replication results from single-SNP and GCDH approaches, we found that pseudo-markers collapsed from pairs of SNPs had a higher replication rate than did individual SNPs (50\% of the loci were replicated for pseudo-markers vs. 35\% for individual SNPs). This can be explained by the much stricter genome-wide significance threshold used in GCDH, i.e., the fewer false positives lead to higher reproducibility.

Although our method provides a higher power to detect $\mathrm{CH}$-like associations, it cannot pinpoint causal variants as does in the conventional GWAS, particularly when the associated SNPs are intergenic. Therefore, our method cannot distinguish between two variants that affect the same or 
different genes and the finding at DMRT2 and SMARCA2 requires further functional studies.

Overall, the GCDH approach seems to be sitting at the saddle point of the two leading theories for "missing heritability": it can detect $\mathrm{CH}$-like interactions between pairs of SNPs often less frequent than what is detectable by the conventional GWAS. For future applications with larger sample sizes and adequate computing power in combination with whole genome-sequencing-based techniques, we expect GCDH to explain a considerably larger percentage of the variance, thus providing a partial explanation for "missing heritability" and new insights into genetics of human complex traits and diseases.

\section{Materials and methods}

\section{The Rotterdam Study}

The Rotterdam Study (RS) is a prospective populationbased data set study of 14,926 participants' aged 45 years and older, living in a suburb of Rotterdam, The Netherlands. Details of the study design and population have been described elsewhere (Hofman et al. 2015). The RS has been approved by the Medical Ethics Committee of the Erasmus MC and by the Ministry of Health, Welfare and Sport of The Netherlands, implementing the Wet Bevolkingsonderzoek: ERGO (Population Studies Act: Rotterdam Study). All participants provided written informed consent to participate in the study and to obtain information from their treating physicians. The RS was conducted according to the Declaration of Helsinki Principles.

Whole blood DNA extraction, genotyping, quality controls, and 1000 Genome (Consortium 2012)-based genotype imputation have been described in detail previously (Kreiner-Moller et al. 2015). In brief, genotyping was carried out using the Infinium II HumanHap550 BeadChips version 3 (Illumina, San Diego, California USA) and Human610-Quad BeadChips. After all quality controls, the current study included a total of 2,543,887 autosomal SNPs (MAF $>0.01$, imputation $R^{2}>0.3$, SNP call rate $>0.95$, and HWE $>1 \times 10^{-3}$ ) in 10,631 individuals. Height was measured using a stadiometer (SECA225; SECA, Hamburg, Germany). We also included 770 extremely tall subject from the Dutch Tall cohort (DT), which used the following inclusion criteria: (1) standard deviation score (SDs) above 1.88 according to Dutch standards (http://www.tno.nl/groei), which corresponds to the $3 \%$ upper tail of the height distribution in Dutch adults, approximately $>195 \mathrm{~cm}$ in men and $>180 \mathrm{~cm}$ in women at age 30, after correcting for secular trend, and (2) Dutch European ancestry defined as being born to Dutch parents who themselves were born in The Netherlands.
The Queensland Institute of Medical Research (QIMR) study

The QIMR study comprised 8672 individuals from predominantly population and family-based studies, around 4161 from nuclear families living in or near Brisbane, Australia, with twins aged around 12-14 years at recruitment for clinical studies, and the remainder from older twin families across Australia recruited through the Australian Twin Registry. Genotyping was a subset of an existing larger data set which used Illumina SNP array chips in large batches from two chip families. Per-batch QC removed SNPs with any of (1) $\mathrm{MAF}<1 \%$; (2) call rate $<95 \%$; (3) HWE $P$ value $<10^{-6}$; (4) mean GenCall score $<0.7$; (5) fail GenomeStudio filters as per Illumina QC SOPs; and (6) blacklists based on poor retest consistency and mapping issues, particularly genderspecific heterozygosity and call-rate filters for chromosome X. Genotyping of the 8672 was divided between firstgeneration Illumina chips (5960 of which 4300 were from $610 \mathrm{~K}$-quad or (rarely) $660 \mathrm{~K}$-quad chips; 1660 from $370 \mathrm{~K}$ or $317 \mathrm{~K}$ chips), and newer Omni and Core + Exome Illumina chips (2712 of which 2279 were from Core + Exome or PsychArray chips; 364 from Omni-2.5; 69 from OmniExpress). Imputation was to the 1000 Genomes Phase 3 mixed-population panel via the University of Michigan's Imputation Server in March-April 2015, as one run per chromosome for each of the two chip families above, in each case for the observed markers available post-QC across all batches for that chip family $(277,690$ markers for first generation and 240,297 markers for Omni/Core + Exome); the two families merged post-imputation with a binary 'run number' covariate in the analysis to mitigate differences in imputation quality. The current study included 4080 unrelated QIMR subjects for replication of the associated SNPs.

\section{GCDH genome screening}

We conducted a genome-wide GCDH screening of adult height for finding $\mathrm{CH}-$ like association in the RS using CollapsABEL. Important parameters of CollapsABEL include the collapsing matrix, the window size, and the p-filter. The collapsing matrix is constructed according to the underlying genetic model, and defines the way that the two SNPs under investigation are collapsed. Assuming a joint recessive model for the minor alleles of two nearby SNPs, one may choose the default collapsing matrix, based on which the two SNPs is collapsed into a pseudo-marker consisting of two possible pseudo-genotypes ( 0 or 1 minor allele vs. 2 or more minor alleles). Alternative collapsing matrices may be of choice when different genetic models are assumed. In the present application of height, we assumed a joint additive effect of the minor alleles of two nearby SNPs and thus used an alternative collapsing matrix, based on which the two 
SNPs under investigation are collapsed into a pseudo-marker consisting of three possible pseudo-genotypes $(0,1$, and 2 or more minor alleles). The difference between compound and double heterozygotes is illustrated in Online Resource 7. It is important to note that compound and double heterozygotes are collapsed into the same pseudo-genotype and thus were not differentiated during the genome screening stage, but were differentiated in the subsequent analysis of the associated regions, where the haplotypes were inferred based on the expectation-maximization algorithm as implemented in the haplo.em function of the $\mathrm{R}$ package haplo.stats (Schaid et al. 2002). When results from haplo.stats show that the $\mathrm{CH}$ group has higher/lower mean height compared to the group with reference alleles in the same direction as $\beta$ values from GCDH analysis, we consider it as a validation of the $\mathrm{CH}$ model. In the absence of $\mathrm{CH}$ group, we consider it an indication of involvement of other genetic mechanisms.

The genotype collapsing is done for all pairs of nearby SNPs within a sliding window which slides over the whole genome with one SNP per step. The size of the sliding window is defined by two criteria, the number of the maximal SNPs $(k)$ and the maximal distance $(d)$ between the index SNP and its pairing SNP. For each window, the first SNP is considered as the index SNP and all the remaining pairing SNPs satisfying both criteria are iteratively collapsed with the index SNP and all collapsed pseudo-markers are iteratively tested for association with the phenotype. Therefore, the maximal number of tests per window is $k$. The minimal $P$ value within each window is assigned to the index SNP. In the current study of height, we used $k=300$ and $d=500 \mathrm{kbp}$, and reported all pairs of SNPs with $P$ values smaller than $1.67 \times 10^{-10}$ (i.e., $5 \times 10^{-8} / 300$ ) from our GCDH genome screening, i.e., Bonferroni correction of the maximal possible number of tests per window.

The p-filter defines the inclusion criteria for SNPs with a minimal marginal effect in the conventional GWAS. In the current study, we set $\mathrm{p}$-filter $=0.1$ to achieve the balance between SNP coverage and the affordable computational burden.

The null distribution of the test statistics from CollapsABEL is expected to be highly inflated, because first, the program only reports the most significant association signal per window, i.e., a problem of multiple testing of correlated SNPs, and second, a p-filter was applied to pre-remove SNPs with little or no marginal effect. Since it was difficult to derive the null mathematically, we conducted a permutation analysis to approximate the null empirically. The whole GCDH genome screening procedure was performed five times using identical parameters, where the genotype-phenotype relationship was broken by randomly shuffling the order of individuals in the phenotype file (height, sex, age, and four PCs) and the resulting test statistics are displayed in QQ plots to illustrate the data-specific null distribution of our GCDH scan for adult height in the RS.

The conventional GWAS for adult height was conducted using PLINK v1.9 assuming an additive allele effect and considering sex, age, and four top genome PCs from the MDS analysis as covariates. Regional Manhattan plots were generated using Locuszoom (Pruim et al. 2010). Power analysis for the QIMR data set was conducted using the pwr $\mathrm{R}$ package (Champely 2013), assuming that $R^{2}$ is consistent across RS and QIMR for all the SNPs tested.

\section{Multivariable and replication analyses}

A previous large-scale meta-analysis of height GWASs identified 697 SNPs at different loci independently contributing to the variation in adult height and 658 SNPs was available in our RS data set. We estimated the proportion of sex- and age-adjusted height variance explainable by these 658 SNPs in our data set by constructing a polygenic risk score based on the weighted allele sums of all 658 SNPs. This polygenic score is referred to below as PRS658. We then accessed the independent effects of PRS658, the SNPs showing genomewide significant association with height in our conventional GWAS, and the pseudo-markers showing genome-wide significant association with height in our GCDH genome screen using a multivariable linear model. More specifically, this model considered sex- and age-regressed height residuals as the dependent variable and included PRS658, the 14 topassociated SNPs from our conventional GWAS, and the 6 top-associated pseudo-markers collapsed from six pairs of SNPs from the GCDH genome screen as the explanatory variables. The multivariable analysis was conducted in an iterative manner to access the $R^{2}$ change due to individual factors using $\mathrm{R}$ scripting, i.e., by adding one independent variable at a time to a linear model in ascending order according to the $P$ values from the multivariable analysis including all independent variables; the $R^{2}$ estimated for the added independent variable then becomes the $R^{2}$ difference between the current model and a previous model. To further compare the height variance explained by the collapsed pseudo-markers and by the individual SNPs, we used sexand age-adjusted residuals as the dependent variable and the collapsed pseudo-marker as the explanatory variable, which is compared with the model using the two individual SNPs of the pair as the explanatory variables.

We conducted a replication analysis for the 14 top-associated SNPs (one SNP per associated region) from the conventional GWAS and the six top-associated pseudo-markers (one per region) from the GCDH genome screen in 4080 unrelated QIMR subjects using linear regression adjusted for sex and age. $P$ values smaller than 0.05 were considered as significant replication. 
Acknowledgements We thank Pascal Arp, Mila Jhamai, Marijn Verkerk, Lizbeth Herrera and Marjolein Peters, PhD, and Carolina Medina-Gomez, MSc, for their help in creating the GWAS database, and Karol Estrada, PhD, Yurii Aulchenko, PhD, and Carolina Medina-Gomez, MSc, for the creation and analysis of imputed data. The authors are grateful to the study participants, the staff from the Rotterdam Study, and the participating general practitioners and pharmacists. KZ is supported by China Scholarship Council to carry out his $\mathrm{PhD}$ studies at Erasmus MC including this work. FL is supported by the Erasmus University Rotterdam (EUR) fellowship and the Chinese recruiting program the "1000-Talents Program" for young scholars. The generation and management of GWAS genotype data for the Rotterdam Study (RS I, RS II, and RS III) was executed by the Human Genotyping Facility of the Genetic Laboratory of the Department of Internal Medicine, Erasmus MC, Rotterdam, The Netherlands. The GWAS data sets are supported by The Netherlands Organization of Scientific Research NWO Investments (nr. 175.010.2005.011, 911-03-012), the Genetic Laboratory of the Department of Internal Medicine, Erasmus MC, the Research Institute for Diseases in the Elderly (014-93-015; RIDE2), and The Netherlands Genomics Initiative (NGI)/Netherlands Organization for Scientific Research (NWO) Netherlands Consortium for Healthy Aging (NCHA), project nr. 050-060-810. The Rotterdam study is funded by Erasmus Medical Center and Erasmus University, Rotterdam, Netherlands Organization for the Health Research and Development (ZonMw), the Research Institute for Diseases in the Elderly (RIDE), the Ministry of Education, Culture and Science, the Ministry for Health, Welfare and Sports, the European Commission (DG XII), and the Municipality of Rotterdam.

\section{Compliance with ethical standards}

\section{Conflict of interest The authors declare no conflict of interest.}

Open Access This article is distributed under the terms of the Creative Commons Attribution 4.0 International License (http://creativecommons.org/licenses/by/4.0/), which permits unrestricted use, distribution, and reproduction in any medium, provided you give appropriate credit to the original author(s) and the source, provide a link to the Creative Commons license, and indicate if changes were made.

\section{References}

Allen HL, Estrada K, Lettre G, Berndt SI, Weedon MN, Rivadeneira F, Willer CJ, Jackson AU, Vedantam S, Raychaudhuri S (2010) Hundreds of variants clustered in genomic loci and biological pathways affect human height. Nature 467:832-838

Bezzina CR, Rook MB, Groenewegen WA, Herfst LJ, van der Wal AC, Lam J, Jongsma HJ, Wilde AA, Mannens MM (2003) Compound heterozygosity for mutations (W156X and R225W) in SCN5A associated with severe cardiac conduction disturbances and degenerative changes in the conduction system. Circ Res 92:159-168

Bodmer W, Bonilla C (2008) Common and rare variants in multifactorial susceptibility to common diseases. Nat Genet 40:695-701

Carlborg Ö, Haley CS (2004) Epistasis: too often neglected in complex trait studies? Nat Rev Genet 5:618-625

Carmichael CM, McGue M (1995) A cross-sectional examination of height, weight, and body mass index in adult twins. J Gerontol A Biol Sci Med Sci 50:B237-B244

Champely S (2013) Package'pwr'. 2015. https://cran.r-project.org/web/ packages/pwr/pwr.pdf. Visited on 04/27/2016

Chan Y, Holmen OL, Dauber A, Vatten L, Havulinna AS, Skorpen F, Kvaløy K, Silander K, Nguyen TT, Willer C (2011) Common variants show predicted polygenic effects on height in the tails of the distribution, except in extremely short individuals. PLoS Genet 7:e1002439

Consortium GP (2012) An integrated map of genetic variation from 1,092 human genomes. Nature 491:56-65

Estrada K, Krawczak M, Schreiber S, van Duijn K, Stolk L, van Meurs JB, Liu F, Penninx BW, Smit JH, Vogelzangs N (2009) A genomewide association study of northwestern Europeans involves the C-type natriuretic peptide signaling pathway in the etiology of human height variation. Hum Mol Genet 18:3516-3524

Hofman A, Brusselle GG, Murad SD, van Duijn CM, Franco OH, Goedegebure A, Ikram MA, Klaver CC, Nijsten TE, Peeters RP (2015) The Rotterdam Study: 2016 objectives and design update. Eur J Epidemiol 30:661-708

Keller MF, Saad M, Bras J, Bettella F, Nicolaou N, Simón-Sánchez J, Mittag F, Büchel F, Sharma M, Gibbs JR (2012) Using genomewide complex trait analysis to quantify 'missing heritability' in Parkinson's disease. Hum Mol Genet 21:4996-5009

Koga M, Ishiguro H, Yazaki S, Horiuchi Y, Arai M, Niizato K, Iritani S, Itokawa M, Inada T, Iwata N (2009) Involvement of SMARCA2/BRM in the SWI/SNF chromatin-remodeling complex in schizophrenia. Hum Mol Genet 18:2483-2494

Kreiner-Moller E, Medina-Gomez C, Uitterlinden AG, Rivadeneira F, Estrada K (2015) Improving accuracy of rare variant imputation with a two-step imputation approach. Eur J Hum Genet 23:395-400. doi:10.1038/ejhg.2014.91

Krude H, Biebermann H, Luck W, Horn R, Brabant G, Gruters A (1998) Severe early-onset obesity, adrenal insufficiency and red hair pigmentation caused by POMC mutations in humans. Nat Genet 19:155-157. doi:10.1038/509

Liu F, Struchalin MV, Duijn K, Hofman A, Uitterlinden AG, Duijn C, Aulchenko YS, Kayser M (2011) Detecting low frequent lossof-function alleles in genome wide association studies with red hair color as example. PLoS One 6:e28145. doi:10.1371/journal. pone. 0028145

Liu F, Hendriks AE, Ralf A, Boot AM, Benyi E, Savendahl L, Oostra BA, van Duijn C, Hofman A, Rivadeneira F, Uitterlinden AG, Drop SL, Kayser M (2014) Common DNA variants predict tall stature in Europeans. Hum Genet 133:587-597. doi:10.1007/ s00439-013-1394-0

Liu F, Hamer MA, Deelen J, Lall JS, Jacobs L, van Heemst D, Murray PG, Wollstein A, de Craen AJ, Uh H-W (2016) The MC1R gene and youthful looks. Curr Biol 26:1213-1220

Mackay TF (2014) Epistasis and quantitative traits: using model organisms to study gene-gene interactions. Nat Rev Genet 15:22-33

Manolio TA, Collins FS, Cox NJ, Goldstein DB, Hindorff LA, Hunter DJ, McCarthy MI, Ramos EM, Cardon LR, Chakravarti A (2009) Finding the missing heritability of complex diseases. Nature 461:747-753. doi:10.1038/nature08494

Marouli E, Graff M, Medina-Gomez C, Lo KS, Wood AR, Kjaer TR, Fine RS, Lu Y, Schurmann C, Highland HM (2017) Rare and low-frequency coding variants alter human adult height. Nature 542:186-190. doi:10.1038/nature21039

Nielen AL, Janss LL, Knol BW (2001) Heritability estimations for diseases, coat color, body weight, and height in a birth cohort of Boxers. Am J Vet Res 62:1198-1206

Ottolenghi C, Veitia R, Barbieri M, Fellous M, McElreavey K (2000) The human doublesex-related gene, DMRT2, is homologous to a gene involved in somitogenesis and encodes a potential bicistronic transcript. Genomics 64:179-186

Ounap K, Uibo O, Zordania R, Kiho L, Ilus T, Õiglane-Shlik E, Bartsch O (2004) Three patients with 9p deletions including DMRT1 and DMRT2: a girl with XY complement, bilateral ovotestes, and extreme growth retardation, and two XX females with normal pubertal development. Am J Med Genet Part A 130:415-423

Pruim RJ, Welch RP, Sanna S, Teslovich TM, Chines PS, Gliedt TP, Boehnke M, Abecasis GR, Willer CJ (2010) LocusZoom: regional 
visualization of genome-wide association scan results. Bioinformatics 26:2336-2337

Ridge PG, Mukherjee S, Crane PK, Kauwe JS (2013) Alzheimer's disease: analyzing the missing heritability. PLoS One 8:e79771

Schaaf CP, Zschocke J, Potocki L (2011) Human genetics: from molecules to medicine. Lippincott Williams \& Wilkins, Philadelphia, Pennsylvania, United States. ISBN:9781608316717, 1608316718

Schaid DJ, Rowland CM, Tines DE, Jacobson RM, Poland GA (2002) Score tests for association between traits and haplotypes when linkage phase is ambiguous. Am J Hum Genet 70:425-434

Seo KW (2007) Dmrt2 and Pax3 double-knockout mice show severe defects in embryonic myogenesis. Comp Med 57:460-468

Silventoinen K, Sammalisto S, Perola M, Boomsma DI, Cornes BK, Davis C, Dunkel L, De Lange M, Harris JR, Hjelmborg JV (2003) Heritability of adult body height: a comparative study of twin cohorts in eight countries. Twin Res 6:399-408

Silventoinen K, Magnusson PK, Tynelius P, Kaprio J, Rasmussen F (2008) Heritability of body size and muscle strength in young adulthood: a study of one million Swedish men. Genet Epidemiol 32:341-349. doi:10.1002/gepi.20308

Tomlinson IP, Carvajal-Carmona LG, Dobbins SE, Tenesa A, Jones AM, Howarth K, Palles C, Broderick P, Jaeger EE, Farrington S (2011) Multiple common susceptibility variants near BMP pathway loci GREM1, BMP4, and BMP2 explain part of the missing heritability of colorectal cancer. PLoS Genet 7:e1002105

Visser M, Kayser M, Palstra R-J (2012) HERC2 rs12913832 modulates human pigmentation by attenuating chromatin-loop formation between a long-range enhancer and the OCA 2 promoter. Genome Res 22:446-455

Visser M, Palstra RJ, Kayser M (2014) Human skin color is influenced by an intergenic DNA polymorphism regulating transcription of the nearby BNC2 pigmentation gene. Hum Mol Genet 23:57505762. doi: $10.1093 / \mathrm{hmg} / \mathrm{ddu} 289$
Visser M, Palstra R-J, Kayser M (2015) Allele-specific transcriptional regulation of IRF4 in melanocytes is mediated by chromatin looping of the intronic rs12203592 enhancer to the IRF4 promoter. Hum Mol Genet 24:2649-2661

Whittock NV, Wan H, Morley SM, Garzon MC, Kristal L, Hyde P, McLean WH, Pulkkinen L, Uitto J, Christiano AM, Eady RA, McGrath JA (2002) Compound heterozygosity for non-sense and mis-sense mutations in desmoplakin underlies skin fragility/woolly hair syndrome. J Invest Dermatol 118:232-238. doi:10.1046/j.0022-202x.2001.01664.x

Wood AR, Esko T, Yang J, Vedantam S, Pers TH, Gustafsson S, Chu AY, Estrada K, Ja Luan, Kutalik Z (2014) Defining the role of common variation in the genomic and biological architecture of adult human height. Nat Genet 46:1173-1186

Yang J, Benyamin B, McEvoy BP, Gordon S, Henders AK, Nyholt DR, Madden PA, Heath AC, Martin NG, Montgomery GW, Goddard ME, Visscher PM (2010) Common SNPs explain a large proportion of the heritability for human height. Nat Genet 42:565-569. doi: 10.1038/ng.608

Yang J, Bakshi A, Zhu Z, Hemani G, Vinkhuyzen AA, Lee SH, Robinson MR, Perry JR, Nolte IM, van Vliet-Ostaptchouk JV (2015) Genetic variance estimation with imputed variants finds negligible missing heritability for human height and body mass index. Nat Genet 47(10):1114-1120

Zhong K, Karssen LC, Kayser M, Liu F (2016a) CollapsABEL: an R library for detecting compound heterozygote alleles in genomewide association studies. BMC Bioinform 17:1-11. doi:10.1186/ s12859-016-1006-9

Zhong K, Verkouteren JA, Jacobs LC, Uitterlinden AG, Hofman A, Liu F, Nijsten T, Kayser M (2016b) Pigmentation-independent susceptibility loci for actinic keratosis highlighted by compound heterozygosity analysis. J Invest Dermatol. doi:10.1016/j. jid.2016.09.007 